\title{
Tratamento endovascular de pseudo-aneurisma de carótida interna em criança
}

\author{
Endovascular treatment of internal carotid pseudoaneurysm in a child
}

\author{
Alexandre Faraco de O liveira ${ }^{1}, \mathrm{D}$ aissuke K ajita ${ }^{1}$, R afael $\mathrm{G}$ omes de Almeida $\mathrm{G}$ arzon ${ }^{2}$, \\ C rescêncio Alberto Pereira Centola 2 , C arla Aparecida Faccio Bosnardo ${ }^{3}$, Miguel Francischelli N eto ${ }^{4}$
}

\begin{abstract}
Resumo
Relato de um caso de paciente feminina, com 6 anos de idade, que apresentou uma massa cervical, dolorosa e com características de processo infeccioso, que revelou ser um pseudo-aneurisma de carótida interna. Foi realizado o tratamento endovascular, com o uso de um balão destacável que determinou a oclusão e conseqüente trombose do pseudo-aneurisma, com excelente resultado.

Palavras-chave: Pseudo-aneurisma, artéria carótida interna, tratamento endovascular.
\end{abstract}

0 pseudo-aneurisma consiste de uma dilatação segmentar do vaso, caracterizada pela perda de continuidadedastrêscamadas da paredearterial naformação do saco aneurismático ${ }^{1}$.

Lesões iatrogênicas e traumáticas levando à formação de pseudo-aneurismas das carótidas não são comuns $^{1}$, principalmente se considerarmos os pacientes pediátricos; mas são de grande importância, tendo em vista 0 al to número de óbitos e seqüelas

1. Médico residente, Serviço de Cirurgia Vascular, Hospital de Ensino, Santa Casa de Limeira, Limeira, SP.

2. Médico radiologista, Serviço de Cirurgia Vascular, Hospital de Ensino, Santa Casa de Limeira, Limeira, SP.

3. Médica assistente, Serviço de Cirurgia Vascular, Hospital de Ensino, Santa Casa de Limeira, Limeira, SP. Coordenadora científica, Programa de Residência Médica, Hospital de Ensino, Santa Casa de Limeira, Limeira, SP.

4. Chefe do Serviço de Cirurgia Vascular e Coordenador dos Programas de Residência Médica em Cirurgia Vascular e Endovascular, Hospital de Ensino, Santa Casa de Limeira, Limeira, SP.

Artigo submetido em 24.11.05, aceito em 13.03.06.

\begin{abstract}
This report is about a 6-year-old female patient who presented a painful cervical mass with characteristics of an infectious process, which was, in fact, an internal carotid pseudoaneurysm. The treatment consisted of the pseudoaneurysm occlusion with an endovascular detachable balloon and its consequent thrombosis, with excellent results.
\end{abstract}

Key words: Pseudoaneurysm, internal carotid artery, endovascular treatment.

incapacitantes, relacionadas com este tipo de doença. Tradicionalmente, o tratamento cirúrgico a céu aberto éo de eleição para estas lesões ${ }^{2}$; contudo, o uso de stents, endopróteses e balões para tratamento endovascular no território carotídeo/vertebral está evoluindo, tornando possível o tratamento das referidas lesões com chance de menor número de complicações relacionadas ao procedimento ${ }^{3}$.

N o caso relatado, além das dificuldades inerentes a qualquer procedimento cirúrgico vascular neste setor, somavam-se outras. T ratava-se de paciente de apenas 6 anos, ea lesão estendia-se até a porção intracraniana da carótida interna.

\section{Caso clínico}

U ma menina de 6 anos de idade foi recebida no serviço de pediatria, apresentando massa cervical à esquerda, com evolução de 10 dias, associada a dor local e febre. Referia ainda amigdalite prévia, tratada com antibióticos. N egava traumas ou punções locais. N ão apresentava histórico médico quanto às internações prévias ou tratamentos anteriores. Ao exame físico, a paciente apresentava massa cervical à esquerda, doloro- 
sa e hiperemiada, sem pulso ou sopro local. U ma tomografia computadorizada cervical revel ou "coleção hipodensa irregular e multisseptada envolvendo o triângulo cervical posterior e região retrofaríngea, estendendo-se abaixo da mastóide até o nível do osso hióide, associada à linfonodomegalia" (Figura 1). A paciente foi, então, levada ao serviço de otorrinolaringologia, que optou por drenagem cirúrgica local. Realizada a cirurgia sem que houvessem intercorrências neste procedimento, não seobservou material purulento, apenas secreção serosa. N o terceiro pós-operatório, a paciente apresentou sangramento em grande quantidade pela ferida operatória que cessou espontaneamente, porém, determinando queda dos níveis de hemoglobina de 10,2 para 6,9. A pósisto, foi chamada a equipe vascular, que solicitou de imediato o duplex cervical, o qual sugeriu pseudo-aneurisma de carótida externa esquerda. A pós este resultado, foi realizada angiografia digital, através de punção da artéria femoral comum direita, passagem de válvula hemostática $5 \mathrm{~F}$, fio guia hidrofílico 0,035 ecateter vertebral $5 \mathrm{~F}$. E videnciou-sepseudoaneurisma de carótida interna, iniciando-se $3 \mathrm{~cm}$ após a bifurcação carotídea e estendendo-se até a porção intracraniana, rechaçando a própria carótida interna e determinando uma lentificação do fluxo em direção ao encéfalo (Figura 2). N o mesmo procedimento, a paciente foi então submetida ao tratamento endovascular através da passagem de válvula hemostática $9 \mathrm{~F}$, passagem de cateter guia $8 \mathrm{~F}$ e posterior passagem, de forma co-axial, de cateter de Sifn e balão destacável gvb 16, liberado no seguimento da carótida interna junto ao colo do pseudo-aneurisma, determinando a oclusão completa da carótida interna esquerda (Figura 3). $\mathrm{Na}$ angiografia de controle, observou-se enchimento do sifão carotídeo via circulação colateral advinda da carótida externa via artéria oftálmica, não havendo, entretanto, enchimento da formação aneurismática sacular.

A pacienteteveótima evolução apóso procedimento, sem qualquer déficit neurológico, sem novos episódios de sangramento e com regressão progressiva do abaulamento. Realizado duplex de controle 7 dias após o procedimento, foi constatada a trombose do aneurisma. No acompanhamento 6 meses após, a paciente manteve-se sem queixas e sem qualquer déficit, com novo duplex inalterado em relação ao anterior.

\section{D iscussão}

O s chamados pseudo-aneurismas, ou aneurismas falsos, comumente necessitam tratamento cirúrgico, pois trazem consigo riscos inerentes de ruptura ou embolização, além desintomas relacionadosà compressão de estruturas adjacentes ${ }^{3,4}$.

U ma das principais causas de pseudo-aneurisma em vasos cervicais é o trauma, mas apenas 0,2\% das admissões de vítimas detrauma determinaram a formação de pseudo-aneurisma da carótida ${ }^{5,6}$.

0 tratamento das lesões das carótidas extracranianas pode se dar tanto cirurgicamente, a céu aberto,

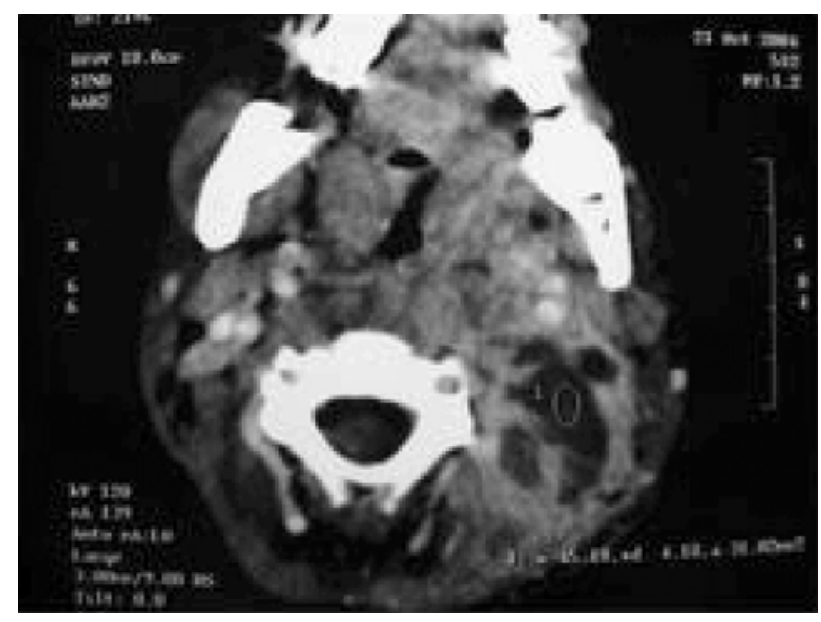

Figura 1 - Tomografia computadorizada pré-operatória, coleção hipodensa irregular e multisseptada, envolvendo o triângulo cervical posterior

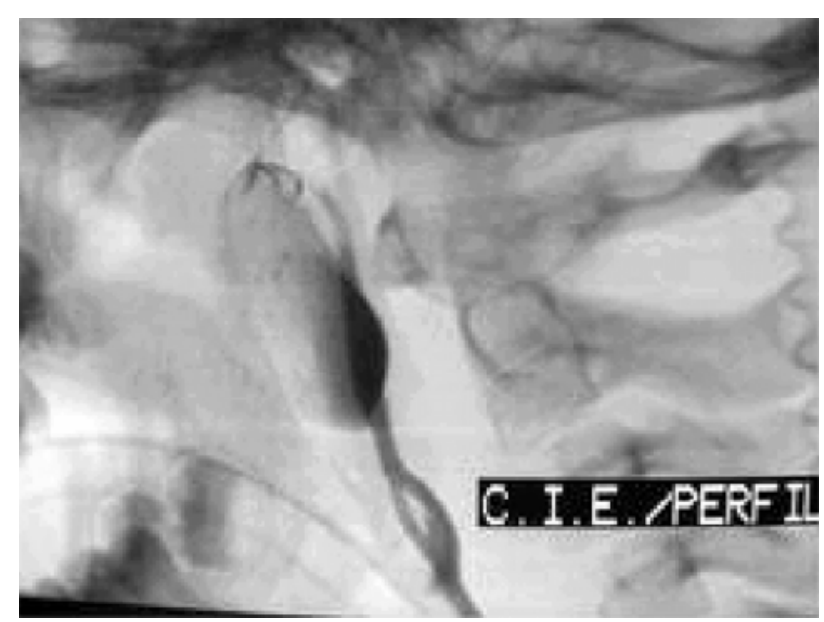

Figura 2 - Pseudo-aneurisma de carótida interna, iniciando-se $3 \mathrm{~cm}$ após a bifurcação carotídea e estendendo-se até a porção intracraniana, rechaçando a própria carótida interna e determinando umalentificação do fluxo em direção ao encéfalo 


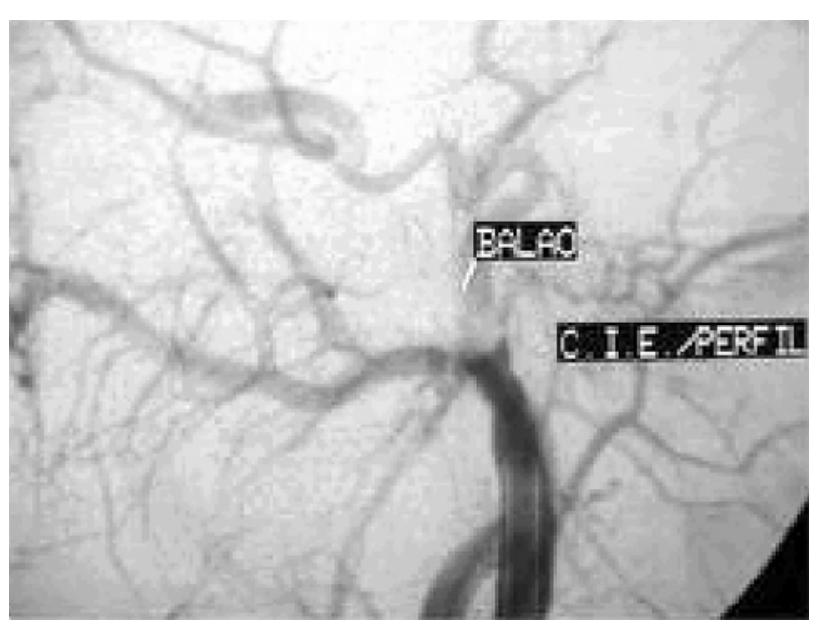

Figura 3 - Balão determinando oclusão completa da carótida interna esquerda

através da ligadura ou reparo com enxerto, como por meios endovasculares, seja através de endopróteses e stents ou por oclusão com molas ou balões $3,7,8$.

As indicações e técnicas para o tratamento cirúrgico a céu aberto de aneurismas e pseudo-aneurismas no segmento carotídeo são conhecidas e tem suas bases determinadas por várias décadas de aplicação ${ }^{2}$. A decisão de utilizar o tratamento endovascular costuma estar associada a condições que dificultem ou impossibilitem o procedimento cirúrgico, como lesões de difícil acesso (junto à base do crânio), em área já abordada, ou com grande risco de ruptura da frágil parede do pseudo-aneurisma?.
$\mathrm{N}$ ão existem, naliteratura, grandes estudosclínicos que mostrem superioridade de um tipo de tratamento em relação a outro, portanto, uma comparação ad equada entre os métodos fica prejudicada. Por um lado, autores como $\mathrm{M}$ ay ${ }^{9}$, que relatou o tratamento endovascular de aneurisma de carótida interna associado a acidente vascular cerebral (AVC) e posterior trombose do stent, consideram o método ainda inadequado para o tratamento de lesões carotídeas?.

Por outro lado, diversos autores $3,6,8-13$ relataram excel entes resultados da terapêutica endovascular, com poucas ou nenhuma complicação, ainda que em séries pequenas (T abela 1$)^{3}$.

Embora não se possa dizer que exista um tratamento endovascular padrão para estetipo de lesão, objetivase utilizar stents ou endopróteses associados ou não à oclusão do aneurisma, de forma a manter a perviedade da artéria acometida pela lesão, promovendo a trombose do aneurisma3,8,10-13.

Coldwell et al. ${ }^{10}$, em uma série de 14 pacientes utilizando stentsisoladamente, obteve sucesso na exclusão completa dos pseudo-aneurismas ${ }^{10}$. Em muitos casos, a colocação isolada do stent pode levar à trombose do pseudo-aneurisma; entretanto, pode ser necessário a interposição de molas através de microcateter entre 0 stent ea parede do pseudo-aneurisma, a fim de promover esta trombose ${ }^{6,8,14}$.

A oclusão intraluminal através de balão pode ser realizada, preferencialmente quando o estudo angiográfico prévio mostre uma circulação cerebral capaz de suprir a área irrigada pela artéria a ser ocluída ${ }^{13}$. Independentedisto, o risco de AV C sempreestará associado ao procedimento 3,7 .

T abela 1 - Resultados do tratamento endovascular de aneurismas e pseudo-aneurismas na carótida ${ }^{3}$

\begin{tabular}{lccc}
\hline Autor & No de pacientes & Tipo de tratamento & Complicações \\
\hline M cN eil et al. ${ }^{6}$ & 1 & Stent recoberto & N enhuma \\
Bush et al. ${ }^{8}$ & 5 & Stent / embolização com molas & 1 AIT \\
M ay et al. ${ }^{9}$ & 1 & Stent recoberto & AVC/trombose stent \\
Coldwell et al. ${ }^{10}$ & 14 & Stent & N enhuma \\
D uke et al. ${ }^{11}$ & 6 & Stent & N enhuma \\
H orowitz et al. ${ }^{12}$ & 2 & Stent & N enhuma \\
Smith et al. ${ }^{13}$ & 1 & Stent recoberto & N enhuma
\end{tabular}

AIT = acidente isquêmico transitório; $\mathrm{AVC}$ = acidente vascular cerebral. 
No caso relatado, tratava-se de paciente do sexo feminino, de 6 anos, cuja artéria carótida interna esquerda, sendo de calibrereduzido, acarretava grande dificuldade na manipulação de stents. T endo sido observado na angiografia diagnóstica a boa patência do sistema carotídeo contralateral e da artéria comunicante anterior, optou-se pela oclusão da carótida interna e, conseqüentemente, do pseudo-aneurisma, acreditando-se na manutenção da perfusão cerebral pela circulação restante. Por fim, esta opção revelouse adequada, tendo a paciente evoluído sem déficits neurológicos.

D esta forma, o tratamento endovascular ganha espaço à medida que novas tecnologias avançam e tendem a trazer soluções menos traumáticas para lesões graves e, principalmente, de difícil acesso para o tratamento cirúrgico a céu aberto.

\section{Referências}

1. Brito CJ, Fonseca Filho VL, Silva RM . Aneurisma arteriais: uma visão geral. In: Brito CJ. Cirurgia vascular: cirurgia endovascular, angiologia. Rio deJ aneiro: Revinter; 2002. vol. 1, p. 432-7.

2. B rito CJ, M erlo I, Fonseca Filho VL. Aneurismas dos troncos supra-aórticos. In: Brito CJ. Cirurgia vascular: cirurgia endovascular, angiologia. Rio deJ aneiro: Revinter; 2002. vol. 1, p. 525-8.

3. M CC ready RA, Divelbiss J L, Bryant M A, D enardo AJ, Scott JA. Endoluminal repair of carotid artery pseudoaneurysms: a word of caution. J V asc Surg. 2004;40:1020-3.

4. H ertz JA, M inion D J, Q uick RC, M ooreEM , Schwartz TH, Endean ED . Endovascular exclusion of a postendarterectomy carotid pseudoaneurysm. Ann V asc Surg. 2003;17:558-61.

5. Ramadan $F$, Rutledge R, O ller D, H owell P, Baker C, K eagy B. Carotid artery trauma: a review of contemporary trauma center experiences. J V asc Surg. 1995;21:46-55.
6. M CN eil JD, Chiou AC, G unlock M G, G rayson D E, Soares G, $H$ agino RT. Successful endovascular therapy of a penetrating zone III internal carotid injury. J V asc Surg. 2002;36:187-90.

7. W yers M C, Powell RJ. M anagement of carotid injuries in a hostile neck using endovascular grafts. J Vasc Surg. 2004;39:1335-9.

8. Bush RL, Lin $P H, D$ odson TF, Dion JE, Lumsden $A B$. Endoluminal stent placement and coil embolization for the management of carotid artery pseudoaneurysms. J Endovasc Ther. 2001;8:53-61.

9. M ay J, White $G H$, W augh $R$, Brennan J. Endoluminal repair of internal carotid aneurysm: afeasiblebuthazardousprocedure. J V asc Surg. 1997;26:1055-60.

10. Coldwell DM, Novak Z, Ryu RK, et al. Treatment of posttraumatic internal carotid arterial pseudoaneurysms with endovascular stents. J Trauma. 2000;48:470-2.

11. Duke BJ, Ryu RK, Coldwell DM, Brega KE. T reatment of blunt injury to the carotid artery by using endovascular stents: an early experience. J N eurosurg. 1997;87:825-9.

12. H orowitz M B, M iller G 3rd, M eyer Y, Carstens G 3rd, Purdy $P D$. U se of intravascular stents in the treatment of internal carotid and extracranial vertebral artery pseudoanerysms. AJN R Am J N euroradiol. 1996;17:693-6.

13. Smith BL, M unschauer CE, D iamond N, RiveraF. Ruptured internal carotid aneurysm resulting from neurofibromatosis: treatment with intraluminal stent graft. J Vasc Surg. 2000;32:824-8.

14. Perez-Cruet MJ, Patwardhan RV, M awad ME, Rose JE. $T$ reatment of dissecting pseudoaneurysm of thecervical internal carotid artery using a wall stent and detachable coils: case report. N eurosurgery. 1997;40:622-5.

Correspondência:

Alexandre Faraco de 0 liveira

Rua Antônio 0 metto, 675, Vila Claudia

CEP 13480-970 - Limeira, SP

Tel.: (19) 3446.6100

E-mail: afaraco@gmail.com 